\title{
Blowup of smooth solutions to the isentropic compressible quantum hydrodynamic model
}

\author{
Jie Zhang ${ }^{1}$, Shu Wang ${ }^{1}$, and fan Geng ${ }^{1}$ \\ ${ }^{1}$ Guangzhou University
}

January 24, 2022

\begin{abstract}
In this article, we consider the blowup phenomenon of smooth solutions to the isentropic compressible quantum hydrodynamic model(QHD) with the initial density of compact support in arbitrary space dimensions. This result is an evolution of Xin's work
\end{abstract}

\section{Hosted file}

Blowup of smooth solutions to quantum hydrodynamic model.pdf available at https:// authorea.com/users/456909/articles/553848-blowup-of-smooth-solutions-to-the-isentropiccompressible-quantum-hydrodynamic-model 